\title{
La investigación educativa y la socialización de resultados en la escuela cubana actual
}

\section{Educational Research and the Socialization of Results in the Current Cuban school}

\author{
Yuliannela Boza-Oramas \\ Dirección de Ciencia y Técnica, Ministerio de Educación \\ http://orcid.org/0000-0001-7749-8861 \\ Cuba
}

\author{
Elmys Escribano Hervis \\ Universidad de Matanzas \\ https://orcid.org/0000-0003-0050-0649 \\ escriba2003@gmail.com
}

Cuba

Mercedes Keeling-Álvarez

Dirección de Ciencia y Técnica, Ministerio de Educación http://orcid.org/0000-0002-8491-5325

\section{Resumen:}

Cuba

El artículo aborda el tema de la significación para el contexto actual de la socialización de resultados de la investigación educativa. El objetivo es argumentar el rol de docentes y directivos educacionales en el proceso de investigación educativa en la escuela cubana. El artículo recoge una elaboración esencialmente teórica; esto exigió la aplicación de métodos teóricos de la investigación, entre ellos el histórico-lógico, el inductivo-deductivo y el análisis documental, lo que ha permitido la determinación del estado de arte contextualizado en la realidad educacional cubana contemporánea. También se hace valer el saber empírico sobre la base de la experiencia de participar en sesiones de análisis del tema en órganos asesores de carácter científico, intercambios sistemáticos con directivos educacionales, docentes y asesores de la Actividad Científica Educacional a nivel de municipios y provincias de Cuba. Se acentúa la necesidad de que directivos y docentes hagan investigación educativa a ciclo completo y socialicen oportunamente sus resultados mediante varios espacios y opciones; esto es un referente para el diseño de políticas en relación al tema.

Palabras clave: Investigación educativa; perfeccionamiento educacional; socialización de los resultados de la investigación.

\begin{abstract}
:
The article addresses the issue of significance for the current context of the socialization of educational research results. The objective is to argue the role of teachers and educational directors in the educational research process in the Cuban school. The article includes an essentially theoretical elaboration; this required the application of theoretical research methods, including the historical-logical, inductive-deductive and documentary analysis, which has allowed the determination of the state of the art contextualized in the educational contemporary Cuban reality. Empirical knowledge is also asserted based on the experience of participating in sessions of analysis of the subject in scientific advisory bodies, systematic exchanges with educational directors, teachers, and advisers of the Educational Scientific Activity at the level of municipalities and provinces in Cuba. The authors highlight the need for managers and teachers to carry out full-cycle educational research and timely socialize their results through various spaces and options; this is a referent for the design of policies regarding the subject.
\end{abstract}

Keywords: Educational research; educational improvement; socialization of research results.

Recibido: 25/02/2021 | Aceptado: 22/04/2021 | Publicado: 07/07/2021

| pág. 1 - 24 
La investigación educativa y la socialización de resultados en la escuela cubana actual

\section{| Introducción}

El Sistema Nacional de Educación Cubano, asume la educación como un proceso complejo que tiene como objetivo preparar al individuo para las exigencias de la vida política, económica y social. Tiene como fin la formación armónica e integral de la personalidad del educando.

En las condiciones actuales, ante los retos que impone el vertiginoso desarrollo científico tecnológico y el cúmulo de información y conocimientos que a diario se suceden, teniendo en cuenta la actualización constante que deben tener los docentes para contribuir al desarrollo integral de los estudiantes, es necesario reflexionar acerca de qué aspectos inherentes a la actividad científica educacional se deben transformar en las instituciones educativas, de forma que se asuma la función investigativa en un nivel superior. Lo anterior, para lograr de manera coherente e integrada, la demostración de la veracidad de los resultados científicos en el aula, institución educativa o provincia en particular, para contribuir a la trasformación educativa.

A la luz del proceso de perfeccionamiento que se desarrolla en el Sistema Nacional de Educación y por la importancia que se le concede a la ciencia, el Instituto Central de Ciencias Pedagógicas [ICCP], 2019, reconoce como funciones:

Propiciar el desarrollo científico y tecnológico, así como la ejecución de investigaciones y servicios científico-técnicos encaminados a la elevación constante de la eficiencia; realizar la divulgación de todos aquellos aspectos que requieran del conocimiento público y generalizado; promover y controlar el proceso de formación y desarrollo efectivo de su potencial científico técnico; garantizar la introducción en la actividad del organismo, de nuevas técnicas y resultados de la ciencia y la innovación tecnológica. 
Para el cumplimiento de estas funciones se considera que la actividad científica es la vía principal para la solución de los problemas que se presentan en la práctica educativa y aporta nuevos conocimientos que enriquecen desde lo teórico y metodológico las Ciencias de la Educación. Entre los autores, que abordan la actividad científica educacional y sus componentes se destacan: Arencibia et al., 2005; Castellanos et al., 2003; Deler, 2006; Escalona, 2008; Hernández et al., 2015; Hernández, 2016; Keeling, 2010; Nocedo, 2015; Reynosa, 2015; Torres, 2016, entre otros.

La actividad científica educacional como proceso sustantivo tiene que estar reflejada en todos los momentos y formas de trabajo del Sistema Nacional de Educación, facilita el proceso de gestión del conocimiento científico pedagógico; orienta la concepción y estructuración de la investigación educativa y permite valorar, criticar 0 asumir las diferentes tendencias de la pedagogía contemporánea.

La tradición educativa nacional cubana, desde sus albores a finales del siglo XVIII ha identificado entre los principales basamentos de la educación la necesidad de estar a la altura de los tiempos y ofrecer respuesta satisfactoria a las urgencias de la sociedad sobre la base de la aplicación de la ciencia, razón por la cual se asumió desde tan temprano momento la cientificidad de la enseñanza y de la escuela. Este pensamiento encontró continuidad en etapas posteriores del desarrollo nacional.

La necesidad de perfeccionar la educación, la escuela y el desempeño de los maestros y los profesores fue una constante en dicha tradición, teniendo en cada etapa de desarrollo, dignos representantes (Chávez, 1996). Con el desarrollo de la educación en el período de la Revolución en el poder se le dio curso satisfactorio a la necesidad de perfeccionar el proceso educativo, perfeccionar la institución educativa y perfeccionar el desempeño del docente y del resto de los profesionales que participan en esta esfera social.

El perfeccionamiento educacional se entiende como el proceso de transformación integraly sostenido de las bases conceptuales, estructurales y organizativas de la educación, 
la escuela y el desempeño de los docentes, en correspondencia con las demandas sociales, el desarrollo de la ciencia y de la tecnología.

Este proceso de cambios y ajustes que implica el perfeccionamiento requiere de una concepción de la Actividad Científica Educacional (ACE) que comprende la investigación educativa concebida y aplicada a nivel de escuela como un elemento dinamizador de la búsqueda de solución a los problemas de la práctica educativa por la vía científica y con la participación activa del docente en dicho proceso.

En Cuba, la ACE se concibe a nivel nacional en correspondencia con la política educacional y la política de desarrollo de la ciencia que establece el Ministerio de Ciencia, Tecnología y Medio Ambiente (CITMA, 2020). Esta se traza a nivel país y debe alcanzar su concreción en las diferentes instituciones educativas, donde tienen lugar los cambios propuestos en el momento actual para el Tercer Perfeccionamiento y que se ponen de manifiesto en el proceso pedagógico dirigido a la formación integral de los estudiantes.
En correspondencia con lo antes expuesto, este artículo se propone como objetivo argumentar el rol de docentes y directivos educacionales en el proceso de investigación educativa en la escuela cubana.

Siguiendo los criterios de Chávez (2001), en la realización del presente artículo se emplearon como métodos científicos los que se mencionan a continuación:

a) Histórico-lógico: para la sistematización de los referentes teórico-metodológicos sobre el tema, así como la ubicación histórica de los mismos, su evolución y manifestación actual en su contexto de relaciones.

b) Inductivo-deductivo: para el procesamiento de resultados de investigaciones sobre actividad científica educacional, en particular, sobre el componente investigación educativa, lo que permitió realizar generalizaciones.

c) Análisis documental: para la revisión de resoluciones, decretos, informes de investigaciones, entre otros, $\mathrm{y}$ 
profundizar en la investigación educativa como proceso y su articulación coherente en el Sistema Nacional de Educación en Cuba.

En el artículo se aborda primero, la actividad científico educacional en el contexto de la escuela cubana, su evolución histórica y la asunción de posiciones teóricas en torno a esta como objeto de interés. En segundo lugar, se fundamenta la concepción de la investigación educativa en la escuela y, por último, se establece la significación de la socialización de los resultados de la investigación educativa.

\section{| Desarrollo}

\section{La actividad científica educacional en la escuela cubana}

La mejor tradición del pensamiento educacional cubano, siempre manifestó confianza en la ciencia, se mantuvo al tanto de sus avances y propendió a que los programas escolares, los textos y las prácticas de los maestros y profesores se identificaran por un claro proceder científico y en vinculación directa con la práctica.
En la primera mitad del siglo XIX José de la Luz y Caballero insistió en la necesidad de que el magisterio, para cumplir con su sagrada misión, se incorpore a la investigación científica del proceso educativo. Decía:

Por medio de la experimentación lograremos acoplar datos en la ciencia didáctica que tanto les ha menester, de la misma manera que se han desarrollado en las físicas, multiplicando el número de colaboradores simultáneos: así se encargarán experimentos y observaciones a los varios maestros al modo de diversos sabios de una misma academia, para que contribuya cada cual con los hechos de su respectivo ramo a la solución de los problemas deseados (De la Luz \& Caballero, 1952, p. 259).

Por su parte, José Martí en la segunda mitad del siglo XIX, se pronunció sistemáticamente por la necesaria cientificidad de la enseñanza y el ejercicio creativo de los maestros vinculado con la vida práctica (Escribano, 2006, 2015).

La concepción de la educación en la Revolución concibió la ciencia como 
un factor importante del proceso de formación. En tal sentido, la Ley 680: Bases y normas legales reguladoras de la Reforma (Ministerio de Educación [MINED], 1959), se pronunció en ese orden.

El impacto de la ACE en el sector educativo está en correspondencia con los avances científicotecnológicos, con las nuevas vías para el acceso a la información y su conversión en conocimiento. Tiene como fin hacer visibles dichos resultados y ponerlos a disposición de los miembros de la comunidad educativa para su introducción y generalización en la práctica educativa y contribuir a su mejoramiento profesional y humano (Boza-Oramas, 2010a; 2015; 2017a; 2017b).

En el Ministerio de Educación de la República de Cuba, esta actividad se define como un "sistema de acciones para la gestión de la investigación, la ciencia, la tecnología y la innovación en el sector educativo. Su dinámica está condicionada por la relación entre sus componentes.” (Escalona, 2008, p. 56).

Dichos componentes son:
- La investigación educativa organizada en programas y proyectos a ciclo completo: es el componente rector de la actividad científica educacional, pues sus objetivos y resultados, confieren cientificidad y sostenibilidad a los procesos de cambio educativo. Para su organización se desarrollan proyectos de investigación que se constituyen en la célula básica para la planificación, organización, ejecución, financiamiento, evaluación y control de actividades vinculadas con la investigación científica, la tecnología y la innovación, así como la formación de recursos humanos.

- La educación de posgrado y formacióndelpotencialcientífico: está dirigida a la formación y preparación de profesionales capaces de resolver problemas por vía científica, innovar de manera creadora y transformar la realidad educativa, a partir de su actualización constante.

- La gestión de la información científica: se manifiesta en el acto de investigación y en el 
vínculo del profesional de la información con los sujetos y con problemas que investigan. Como proceso subjetivo, la evolución de la gestión de información se hace evidente por los sucesivos acercamientos

a nuevos conocimientos.

- La socialización, publicación y reconocimiento de resultados científicos: promueve el intercambio de los resultados de la ciencia entre los sujetos que intervienen en la actividad científica; permite la comunicación de la ciencia, la divulgación, publicación y reconocimiento de los resultados de la investigación educativa.

La ACE se materializa en vías, formas $y$ acciones pedagógicas para su gestión y socialización; se trata de un enfoque de la ciencia como actividad. De ahí la importancia de perfeccionarla sistemáticamente como herramienta de dirección en las instituciones educativas, para que las principales transformaciones educacionales se ajusten a la dinámica de los procesos socioeducativos que en ellas tienen lugar.
Los objetivos estratégicos de esta actividad en el Ministerio de Educación han quedado definidos como:

- Propiciar, mediante la investigación, el adecuado desarrollo de los procesos de cambio educativo que permitan elevar la calidad de la educación en los diferentes niveles educativos de la escuela cubana en las condiciones actuales y perspectivas, así como producir los fundamentos teóricos $\mathrm{y}$ metodológicos que los sustenten.

- Convertir la actividad científica educacional en la vía de identificación y búsqueda de solución de los problemas educacionales en cada provincia, a partir de la integración del potencial científico a los proyectos, bajo la dirección del personal más calificado y la intervención directa de directivos, investigadores, docentes, trabajadores, estudiantes y profesionales de otros organismos vinculados a la educación.

La ACE ha estado signada por las exigencias $y$ demandas sociales 
que se expresan en el contexto educativo cubano, por los cambios y proyecciones presentes en los diferentes perfeccionamientos que han tenido lugar en el Sistema Nacional de Educación. En este sentido, se hace alusión al hecho de que en 1975 se dictó la Tesis de Política Educacional, con su correspondiente Resolución, que oficializaba a la pedagogía con un carácter socialista marxistaleninista y reforzaba el enfoque humanista y martiano. En dicha declaratoria se precisó el fin y los objetivos de la educación.

En esta fecha se concretó y aprobó el Primer Perfeccionamiento del Sistema Nacional de Educación, como resultado de un estudio científico minucioso. Su puesta en práctica hasta 1985, significó un cambio de estructura de los subsistemas que lo conforman y en los contenidos de los planes y programas de estudio, así como en la elaboración de libros de texto y demás materiales escolares, teniendo en cuenta los avances científico tecnológicos.

Entre 1986 y 1990 se realizó el Segundo Perfeccionamiento del Sistema Nacional de Educación.
En esta ocasión, se proyectó la modernización y flexibilización de los planes y programas de estudio. Se realizó un profundo estudio de carácter comparado acerca del estado de la educación en diferentes países y con esta experiencia concebir la educación de la mejor manera en correspondencia con los intereses y necesidades del país. Se estimuló la realización de investigaciones para el desarrollo de habilidades intelectuales con un sustento esencialmente psicológico.

En el 2014 comienza la implementación del Tercer Perfeccionamiento del Sistema Nacional de Educación, dirigido al igual que los anteriores, por el Instituto Central Ciencias Pedagógicas. En esta ocasión, tiene un enfoque integral y multifacético debido a la naturaleza compleja de los problemas presentes en la educación. En dicho proceso se emplea de forma sistémica la ciencia y se acompaña de la sistematización e introducción de los resultados científicos.

Entre los factores que han determinado la necesidad de acometer el Tercer Perfeccionamiento del 
Sistema Nacional de Educación se encuentran:

- Los cambios operados en la sociedad a escala mundial y nacional, así como el desarrollo científico-tecnológico.

- Necesidad de flexibilizar y contextualizar los estilos de dirección, el currículo, el trabajo de las instituciones $y$ modalidades educativas.

- Fortalecer la preparación de directivos y docentes desde la incorporación de la investigación científica como una de las vías principales para dar solución a los problemas que se presentan en la práctica educativa, así como la incorporación de los resultados científicos como herramienta pedagógica en la preparación continua y sistemática de los docentes de todos los niveles educativos.

En este Perfeccionamiento se proponen dos cambios fundamentales, dirigidos a las formas de trabajo y a cambios curriculares. En particular, los cambios en las formas de trabajo son:

- El Proyecto Educativo Institucional y de Grupo.

- La forma de dirigir el trabajo en la institución educativa.

- Participación de los distintos factores del centro y de la comunidad.

- El Trabajo en Red.

- La permanente consulta con los distintos factores de la sociedad.

- El empleo de la experimentación.

- La sistematización e introducción de los principales resultados científicos.

Los cambios curriculares incluyen el currículo general e institucional. Se proponen la materialización de las concepciones educativas al interior de los programas; actividades y programas complementarios, proyectos técnicos sociales, círculos de interés, sociedades científicas, entre otras.

Se puede destacar que, en los tres momentos de perfeccionamiento del Sistema Nacional de Educación, 
la actividad científica ha sido concebida para lograr los resultados esperados en correspondencia con el fin de la educación cubana; en la producción de nuevos conocimientos, la concepción de la pedagogía cubana asumida y en la participación de los docentes, directivos e investigadores en las propuestas realizadas.

La filosofía implícita en la concepción de este Tercer Perfeccionamiento, reclama mayor responsabilidad de la institución educativa y de sus docentes en la conformación del proyecto educativo institucional y el currículo institucional. "La concepción curricular para el perfeccionamiento expresa la necesaria consideración de un currículo con un carácter integral, flexible, contextualizado y participativo" (ICCP, 2016, p. 1).

Esta proyección implica que docentes y directivos apliquen de forma sistemática métodos, técnicas e instrumentos de investigación para conocer con mayor profundidad la familia de los escolares que acceden a la institución educativa, para conocer también la comunidad en que está enclavada su escuela y propender al aprovechamiento de las potencialidades que en el orden cultural, científico, tecnológico, deportivo, etc. puede ofrecer al fortalecimiento del currículo institucional y su proyecto educativo. Esta concepción se alcanza con docentes y directivos que puedan aplicar la investigación educativa como un elemento dinamizador de gestión de la calidad.

Entre los principios para el Tercer Perfeccionamiento del Sistema Nacional de Educación se expresa que:

La investigación pedagógica, es uno de los instrumentos de carácter científico que ocupa un importante lugar en las tareas de perfeccionamiento del sistema, pues la formación integral de las nuevas generaciones ha de ser el resultado de la óptima planificación y dirección científica de dicho proceso. (ICCP, 2019, p. 33)

De ahí que la ACE se proyecta en este perfeccionamiento con un sentido estratégico de dirección de la ciencia mediante la: 
- Consolidación de los proyectos institucionales como el eje articulador de la ACE en las instituciones educativas.

- Precisión en los encargos que se hacen en cada provincia a los proyectos de investigación asociados a programas nacionales.

- Identificación de líderes científicos en los diferentes niveles educacionales, que pueden asumir tareas de dirección de esta actividad.

- Coherencia entre los resultados de la investigación obtenidos en proyectos de investigación, los eventos que se realizan y las publicaciones que se acreditan.

- Estimulación y asesoramiento a docentes, investigadores y directivos para el uso eficiente de las posibilidades de premios y reconocimientos que se otorgan dentro y fuera del organismo.

Ello ha requerido que los docentes se involucren desde su desempeño en la actividad de investigación científica, la que, de acuerdo con Chávez (2007), se caracteriza porque:
- Partir de problemas, necesidades y contradicciones de la práctica.

- Tiene carácter intencional y consciente.

- Es un proceso metódico, que se realiza apoyado en métodos, técnicas y procedimientos teóricos, empíricosy matemáticos estadísticos.

- La búsqueda del conocimiento se fundamenta en referentes teóricos y los resultados dan respuesta a las demandas sociales y enriquecen la teoría.

- La información obtenida permite describir hechos y predecir la realidad.

- El fin último de la actividad científica es la práctica, donde la teoría, además de ser contrastada, contribuye al mejoramiento de la calidad de vida.

Dicha actividad se materializa desde el proceso de investigación educativa, considerada su componente rector. Sus objetivos y resultados confieren cientificidad y sostenibilidad a los procesos de cambio educativo. Para su organización se desarrollan proyectos de investigación. 
II. Concepción de la investigativo como parte esencial de investigación educativa en la la estrategia metodológica que utilice escuela

La escuela es el centro principal de formación y transmisión de conocimientos, saberes y valores, donde tiene lugar el acto de enseñanza-aprendizaje para el logro de la calidad de la educación. Confluyen en esta el colectivo de estudiantes, claustro de profesores y otros agentes y agencias educativas, que desde sus roles potencian el proceso pedagógico.

En el contexto del salón de clases, la escuela y su relación con la familia y la comunidad se manifiestan problemáticas que requieren ser investigadas y deben ser asumidas por el docente en su propio radio de acción. Ello implica concebir al maestro más allá de su función docente-educativa, debe ser visto en su desempeño profesional como un investigador.

Según señala Chávez (2007), el docente como investigador puede asumir la investigación educativa como colaborador de un equipo de investigación especializado; como investigador directo de su propio proceso de enseñanza-aprendizaje; $\mathrm{y}$, como aplicador del método para el desarrollo del contenido del programa que imparte.

El hecho de que el principal gestor del proceso educativo en la institución escolar se desempeñe con una filosofía propia del método de la ciencia, constituye una potencialidad o fortaleza para cualquier sistema educativo. Ello propicia la determinación y jerarquización de los problemas que se pueden transformar de forma sostenida mediante la aplicación de la ciencia, para trabajar de forma participativa en la aplicación de la ciencia en función de la solución (Boza-Oramas, 2010b, 2016).

Entre las principales vías que desde la escuela se emplean para dar respuesta a los procesos formativos se encuentran las experiencias pedagógicas de avanzada y la investigación educativa.

Las experiencias pedagógicas de avanzada son el "proceso consciente de búsqueda de conocimientos sobre los problemas de la práctica pedagógica escolar, con métodos de investigación de tipo empírico fundamentalmente ... no exige una elaboración teórica 
profunda y su ejecución está asociada a la práctica del aula”. (ConcepciónGarcía \& Rodríguez-Expósito, 2004, p. 2).

La investigación educativa se define como el:

proceso dialéctico de construcción del conocimiento científico multidisciplinar acerca de la realidad educativa, conscientemente orientado y regulado por el método científico, con la finalidad de producir determinados resultados científico-técnicos que posibilitan describir, explicar, predecir y transformar el objeto, en correspondencia con los problemas inmediatos y perspectivos del desarrollo de la educación en un contexto histórico concreto. (Castellanos et al., 2003, p. 29).

La primera forma parte de la propia dinámica de los modos de actuación de docentes y directivos, mientras que la segunda ha ido cobrando mayor intencionalidad a partir de la Maestría en Ciencias de la Educación de Amplio Acceso, las transformaciones $\mathrm{y}$ proyecciones del Tercer Perfeccionamiento y el reordenamiento del Sistema de Ciencia, Tecnología e Innovación cubano.

Aunque los autores de este artículo reconocen las diferencias entre estas, que se presentan en la Figura 1:

Figura 1.

Diferencias esenciales entre la investigación educativa y la experiencia pedagógica de avanzada.

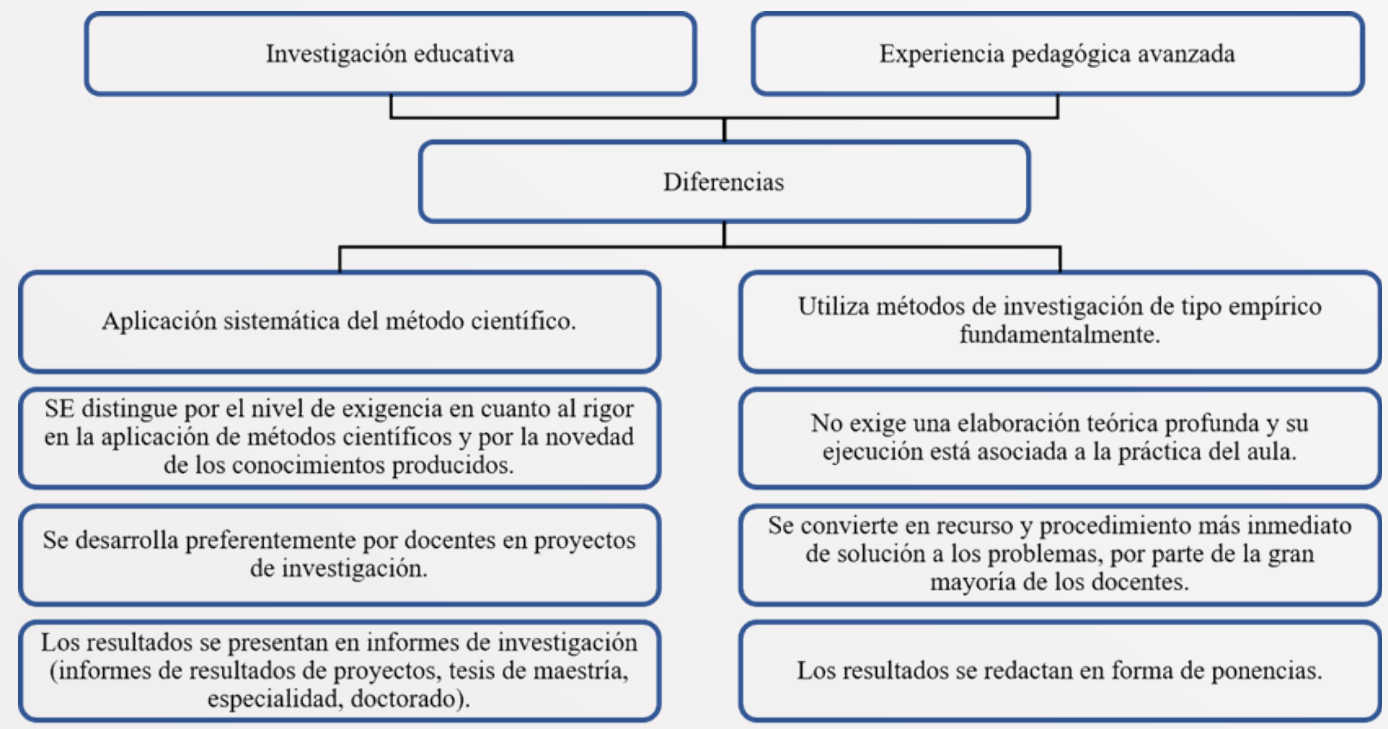

Nota: Elaboración propia. 
Mientras tanto, se ponderan como semejanzas:

- Procesos conscientes de construcción y búsqueda de nuevos conocimientos.

- Estudian los problemas de la realidad educativa y aportan conocimientos $\mathrm{y}$ experiencias novedosas para su transformación positiva.

- Permiten

un mejor entendimiento de las realidades, brindan nuevas perspectivas que suceden alrededor del ámbito educativo.

- Participación activa del docente en la búsqueda de solución a los problemas de su práctica en su contexto de actuación.

- Favorecen una actitud científica y valores investigativos en los docentes.

- Aportan resultados significativos para el desarrollo de la educación y la gestión continua de su calidad.

- Son una vía expedita para desarrollar experiencias creativas y de innovación necesarias en los contextos escolares y docentes.

En esta concepción de la investigación educativa se parte del reconocimiento de la escuela como laboratorio principal para determinar las potencialidades y debilidades del proceso de enseñanza-aprendizaje. En ella se debe identificar el banco de problemas existentes y de estos, seleccionar las prioridades, que se constituyen en líneas de investigación. De las problemáticas detectadas se fijan las que requieren de la ciencia para su solución.

Otro de los aspectos que tratan los investigadores tiene que ver con la índole de la investigación sobre educación y en la educación, así como la afirmación de que el compromiso ético de directivos y docentes es influir, por medio de los procesos y resultados de su trabajo, en la mejora de la educación en general, en la práctica educativa $\mathrm{y}$, por supuesto, en la teoría que se sustenta en un momento histórico determinado.

Se considera al docente como el principal investigador de su propia práctica con el desarrollo del 
proceso de investigación educativa y de las experiencias pedagógicas de avanzada. Destacando el papel activo de este, al decir de Fernández et al. (2005):

El investigador no es un colector de datos, sino un sujeto activo implicado en un proceso de relación con el otro ... El investigador, en su carácter activo y pensante, es el instrumento esencial de la investigación. El papel activo del investigador se expresa en las decisiones que tiene que asumir en el curso de la investigación; $\mathrm{y}$ estas tienen consecuencias únicas e irreversibles para el desarrollo del conocimiento. El investigador no es un usuario de un conocimiento producido fuera de él a nivel instrumental, sino el sujeto de este proceso dentro del cual están implicadas todas sus acciones y decisiones (p.199).

La investigación educativa tiene el compromiso social de producir buenas prácticas desde la reflexión consciente, pues necesariamente debe buscar la mejora de la educación y con la mejora de este importante proceso y resultado, el perfeccionamiento de la sociedad. También la catalogan como una estrategia de producción de nuevos conocimientos de diversos niveles de rigurosidad y alcance para indagar y conocer la práctica, para intervenirla e innovarla o para aportar a la educación, a la política educativa o a la toma de decisiones desde la práctica misma.

En la concepción de la investigación educativa en el contexto escolar, la introducción en la práctica de los resultados científicos, es un momento de validación de dicha investigación. Su implementación y seguimiento permite valorar los cambios operados. Por otra parte, se concibe la necesaria socialización y publicación de los resultados obtenidos con el empleo de diversas formas de trabajo metodológico, superación e investigación.

Desde los proyectos de investigación debe concebirse la forma más idónea para socializar y reconocer los resultados científicos, teniendo en cuenta la naturaleza de estos. Puede ser a través de ponencias para eventos científicos, libros electrónicos o impresos, folletos, artículos, multimedias, entre otros. 


\section{Significación de la socialización de los resultados de la investigación educativa}

Una investigación es válida y reconocida como tal si existe un documento o fuente (en soporte papel o electrónico) que se pone a disposición y se difunde entre los miembros o integrantes de la comunidad científica. Debe contener información suficiente para entender aportes, evaluar resultados, transferirlos, originar nuevas investigaciones y resultados y elevar el nivel de la ciencia. Pero debe, además regirse por normas editoriales de formato, de referenciación y citación de fuentes bibliográficas.

Los aspectos antes señalados también forman parte de la concepción de investigación educativa en el contexto cubano y que se manifiesta en la socialización, publicación y reconocimiento de estos.

La socialización es considerada el resultado que se debe alcanzar, que asume como elemento clave el proceso de mediación de los sujetos que intervienen en la actividad científica educacional. Favorece en los docentes, directivos e investigadores, el intercambio, transmisión, identificación, registro y apropiación de la información y el nuevo conocimiento, resultados de la actividad científica que llevan a cabo de forma individual y colectiva (Boza-Oramas, 2018a; 2018b; BozaOramas \& Keeling-Álvarez, 2019).

En la socialización se reconoce la publicación como una de las vías esenciales para dar a conocer los resultados de la investigación educativa. Esta no responde solo al resultado final, sino a los resultados parciales que se van obteniendo en el proceso investigativo, que permite obtener criterios, valoraciones y sugerencias de otros investigadores que enriquecen el resultado final.

Se realiza mediante las diferentes fuentes y recursos de información, principalmente las revistas científicas. Unido a ello está el reconocimiento de estos resultados, visto como la acción y efecto de distinguir a los docentes, investigadores, instituciones $\mathrm{y}$ sus resultados investigativos, que promuevan la solución de los problemas que se presentan en la 
práctica educativa y que pueden ser introducidos y generalizados en el contexto de la educación.

La socialización, publicación y reconocimiento de resultados científicos es el componente de la actividad científica educacional que permite promover condiciones y relaciones sociales para la comunicación de la ciencia. Existen diferentes vías reconocidas en el Sistema Nacional de Educación cubano para la socialización de resultados científicos, entre las más reconocidas se encuentran: la realización sistemática de eventos científicos y espacios de presentación de la investigación educativa; la organización específica de talleres de generalización de resultados científicos. También habría que mencionar por su importancia los movimientos de integración profesional y científica (Asociación Nacional de Innovadores y Racionalizadores, Fórum de Ciencia y Técnica y Brigadas Técnicas Juveniles). Al mismo tiempo, también resultan de interés las exposiciones sobre los logros alcanzados por la educación cubana en diferentes ámbitos, principalmente los resultados de la actividad científica educacional; ejemplo de ello la exposición anual en el Pabellón de Educación en el Recinto Ferial Expocuba. Se asume también, sobre todo en las instituciones educativas, el trabajo científico-metodológico. Existen publicaciones que tributan a la socialización, visibilidad e impacto del investigador y sus resultados científicos; y, por último, se menciona la Enciclopedia Colaborativa Cubana EcuRed de creciente uso en la educación cubana (Boza-Oramas, 2018a; 2018b).

Los profesionales de la educación necesitan estar actualizados y para ello deben participar del debate científico contemporáneo y exponer de manera sistemática sus resultados y experiencias ante la comunidad científica nacional e internacional. Las Tecnologías de la Información y las Comunicaciones abren nuevas vías de forma creciente para el acceso, la conservación y la socialización de los resultados de la investigación educativa (Vienni, 2014).

Una vía para ello se encuentra hoy en el acceso a miles de revistas digitales en el mundo, las cuales permiten por una parte el acceso a información actualizada en diversos temas de 
interés, y por otra parte, son una plataforma para socializar de forma rápida y directa el resultado de las experiencias de avanzada y los resultados de las investigaciones en el ámbito de la institución educativa y los procesos que en la misma se gestan.

Otra vía es la participación en eventos, ya que las condiciones de distanciamiento sanitario impuestas por la pandemia por COVID-19 han propiciado de forma acelerada la concepción de los mismos por parte de instituciones y redes profesionales desde espacios virtuales.

En el caso de Cuba, un ejemplo de ello es el Congreso Internacional Pedagogía, el cual se ha consolidado como el evento más importante y masivo del Ministerio de Educación (MINED). Para la edición del 2019, en el proceso nacional preparatorio, se realizaron 8 mil 303 eventos a nivel de institución educativa, participaron 242 mil 989 docentes, de ellos 15 mil 616 son directivos y se presentaron 85 mil 127 trabajos. Lo anterior, según datos de la Dirección de Ciencia y Técnica del MINED, que tiene a su cargo la organización de dicho movimiento desde las instituciones educativas a nivel de base hasta el Congreso. Los cursos precongresos han permitido la socialización, publicación y reconocimiento de resultados científicos, que son resultados de proyectos de investigación que involucran a diferentes instituciones, provincias, como expresión del carácter interdisciplinario y complejo de los problemas de la práctica educativa, cuya solución requiere de dicho enfoque. Este evento se realizó de manera virtual con participación internacional en febrero de 2021.

Resulta de gran importancia la concepción de la Actividad Científica Educacional que, en el caso de Cuba, rige de forma coherente el desarrollo de la investigación educativa dentro del Ministerio de Educación y como parte de dicha concepción se correlaciona con el necesario proceso de socialización de los resultados más destacados de este tipo específico de actividad científica.

\section{| Conclusiones}

La ciencia y sus resultados científicos aportan a la teoría y a la metodología del área del saber a la que tributan, pero sobre todas las cosas, tienen 
un compromiso social, de ahí que deben ser del dominio de todos los interesados, lo que exige el diseño de estrategias que contribuyan a su socialización y el compromiso individual y colectivo de los investigadores, las instituciones $\mathrm{y}$ organismos.

La tendencia mundial prevaleciente se orienta a la integración de la producción del conocimiento científico en la planificación de las políticas públicas de desarrollo social. Por tanto, los resultados de la actividad científica en el campo educacional desempeñan un creciente papel en la toma de decisiones, la solución de los problemas inmediatos y perspectivos y la construcción de la teoría, como guía indispensable para toda acción transformadora.

En Cuba la concepción de la Actividad Científica Educacional es un proceso sistemático de trabajo para el empleo de la ciencia como un modo de transformar la realidad educativa y contribuir a la calidad de la educación. Uno de sus pilares esenciales es considerar al maestro como investigador. El educador debe emplear el método de la ciencia para atender y solucionar las diferentes problemáticas de carácter pedagógico-educativo que se manifiestan en su contexto de desempeño profesional, especialmente la clase, la escuela y la relación de esta con la familia, las organizaciones y las instituciones de su comunidad.

La investigación educativa realizada por docentes y directivos de las diferentes instituciones educativas, aporta distintos tipos de resultados científicos que dan solución a los problemas de la práctica educacional y su introducción permite la transformación de esta.

Una adecuada concepción sobre las diferentes formas de socialización de los resultados de la investigación educativa, que incluya espacios para el intercambio profesional en eventos -en la actual coyuntura preferenciando la modalidad virtual-, la publicación en libros y revistas, y el justo reconocimiento de los resultados científicos más destacados, es componente de la 
Actividad Científica Educacional. intercambios, divulgación y Esto propicia la promoción de reconocimiento de los resultados de mejores condiciones y relaciones dicha actividad, contribuyentes a la para la comunicación, publicación, mejora de la calidad educativa. 
La investigación educativa y la socialización de resultados en la escuela cubana actual

\section{|Referencias}

Arencibia, V., Hernández, R., Llivina M. \& Valdés O. (2005). Proyectos de investigación educativa: una alternativa en la gestión de la actividad científica. Instituto Superior Pedagógico "Enrique José Varona”.

Boza-Oramas, Y. (2010a). Actividad Científica Educacional. Principales regularidades a partir del análisis de los componentes que la integran. Organismos de la Administración Central del Estado del Congreso Internacional Pedagogía. Editorial Universitaria.

Boza-Oramas, Y. (2010b). Investigación educativa y formación investigativa de profesionales de la Educación. Congreso Internacional Educación Superior Universidad. Editorial Universitaria.

Boza-Oramas, Y. (2015). La interdisciplinariedad en la actividad científica educacional. Congreso Internacional Pedagogía. Órgano Editor Educación cubana.

Boza-Oramas, Y. (2016). La interdisciplinariedad en la investigación de los profesionales de la educación. Congreso Internacional de Educación Superior Universidad. Editorial Universitaria.

Boza-Oramas, Y. (2017a). La socialización de resultados de investigación en la práctica educativa. Retos actuales. Congreso Internacional Pedagogía. Órgano Editor Educación cubana.

Boza-Oramas, Y. (2017b). La actividad científica educacional como proceso continuo de desarrollo en el marco de las actuales transformaciones educacionales. Principales resultados y perspectivas de desarrollo. Congreso Internacional Pedagogía. Órgano Editor Educación cubana.

Boza-Oramas, Y. (2018a). Estrategia metodológica para potenciar la socialización de resultados científicos en la formación docente. Congreso Internacional de Educación Superior Universidad. Editorial Universitaria. 
Boza-Oramas, Y. (2018b). Socialización, visibilidad e impacto de los resultados de la investigación educativa. Organismos de la Administración Central del Estado del Congreso Internacional Pedagogía. Editorial Universitaria.

Boza-Oramas, Y., \& Keeling-Álvarez, M. (2019). La socialización de los resultados de la investigación educativa. Revista Pedagogía Profesional, 17(1).

Cáceres, G. (2014). La importancia de publicar los resultados de Investigación. Facultad de Ingeniería, 23(37), 7-8. http://bitly.ws/bVbL

Castellanos, B., Fernández, A.M., Llivina, M.J., Arencibia, V., \& Hernández, R. (2003). La gestión de la actividad de ciencia e innovación tecnológica y la competencia investigativa del profesional de la educación. IPLAC.

Chávez, J.A. (2007). La investigación científica desde la escuela. ICCP.

Chávez, J. A. (2001). Apuntes para una metodología de la investigación educativa. Universidad Autónoma de Guerrero.

Chávez, J.A. (1996). Bosquejo histórico de las ideas educativas en Cuba. Pueblo y Educación.

Concepción-García, M.R., \& Rodríguez-Expósito, F. (2004). Metodología de la experiencia pedagógica de avanzada. Ciencias Holguín, Revista trimestral, 10, 2-9. http://bitly.ws/bVbN

De la Luz y Caballero, J. (1952). Escritos Educativos. Universidad de la Habana.

Deler, G. (2006). Estrategia para la dirección de la actividad científicoinvestigativa del docente en el centro de referencia de la educación primaria. [Tesis doctoral, Instituto Central de Ciencias Pedagógicas].

Escalona, E. (2008). Estrategia de introducción de resultados de investigación en el ámbito de la actividad científica educacional. [Tesis doctoral, Instituto Central de Ciencias Pedagógicas].

Escribano, E. (2006). La concepción de la educación en la obra de José Martí. Editorial Pueblo y Educación. 
Escribano, E. (2015). El fomento de la Cultura de la Naturaleza, desde el pensamiento de José Martí. Integra Educativa, 8(3), 87-99.

Fernández, A.M., Castellanos, B., Llivina, M.J., Arencibia, V., \& Hernández, R. (2005). La investigación educativa desde un enfoque dialéctico. IPLAC.

Keeling, M. (2010). Concepción teórico-metodológica para favorecer el desarrollo de la actividad científica educacional de docentes $e$ investigadores desde el centro de documentación e información pedagógica de la Universidad de Ciencias Pedagógicas Enrique José Varona. [Tesis doctoral, Universidad de Ciencias Pedagógicas "Enrique José Varona”].

Hernández, Y. (2016). Concepción de dirección para la gestión científica pedagógica en la Dirección Provincial de Educación de La Habana. [Tesis doctoral, Instituto Central de Ciencias Pedagógicas].

Hernández, M.A., Chirino, M.V., \& Hernández, E. (2015). La introducción de resultados investigativos, un problema de actualidad en la formación del Ingeniero en Ciencias Informáticas. Revista Cubana de Ciencias Informáticas, 9(4), 142 -153. http://bitly.ws/bVbR

Instituto Central de Ciencias Pedagógicas [ICCP]. (2019). Documentos para el perfeccionamiento del Sistema Nacional de Educación. ICCP.

Instituto Central de Ciencias Pedagógicas [ICCP]. (2016). Ideas preliminares para la construcción del currículo institucional en el marco del perfeccionamiento de los niveles educativos del Ministerio de Educación. ICCP.

Ministerio de Ciencia, Tecnología y Medio Ambiente [CITMA]. (2020). Indicaciones Metodológicas para la actividad de Programas y Proyectos de Ciencia, Tecnología e Innovación. Editorial La Habana.

Ministerio de Educación [MINED]. (1959). Ley 680: Bases y normas legales reguladoras de la Reforma. MINED. 
Nocedo, I. (2015). Estrategia de gestión para la introducción de resultados de investigación en la Educación Primaria. [Tesis doctoral, Universidad de Ciencias Pedagógicas “Enrique José Varona”].

Reynosa, M. (2015). La organización de la actividad científica educacional en una institución de la Educación Preuniversitaria. [Tesis doctoral, Universidad de las Tunas].

Torres, P. (2016) Retos de la investigación educativa cubana actual: Aportes a su tratamiento. [Tesis doctoral, Instituto Central de Ciencias Pedagógicas].

Vienni, B. (2014). La socialización del conocimiento científico como problema interdisciplinario. [Tesis doctoral, Universidad de Granada]. http://hdl.handle.net/10481/34051 